\title{
TWO ELEMENT GENERATION OF THE SYMPLECTIC GROUP1
}

\author{
P. F. G. STANEK
}

Communicated by Irving Kaplansky, November 7, 1960

Albert and Thompson [1] have given two generators for the projective unimodular group over any finite field, one of which has period (group order) two. Using the same method, it is possible to prove a similar result for the symplectic group, which may be described as the group of linear transformations on an even-dimensional vector space which leave invariant a skew-symmetric bilinear form.

Let

$$
H=\left(\begin{array}{rr}
0 & I \\
-I & 0
\end{array}\right)
$$

be a $2 n$ by $2 n$ matrix and let $G(2 n, q)$ be the group of all matrices $X$, with entries from $G F(q)$, which satisfy $X H X^{T}=H$, where $X^{T}$ is the transpose of $X$. Denote by $S(2 n, q)$ the group $G(2 n, q)$ modulo its center. $S(2 n, q)$ is known to be simple, except for $n=1, q=2$ or 3 , and $n=2, q=2$.

The following three types of matrices are known to be generators of $G(2 n, q)$ (see [2]):

(i) translations:

$$
T=\left(\begin{array}{cc}
I & S \\
0 & I
\end{array}\right), \quad \text { where } \quad S^{T}=S
$$

(ii) rotations:

$$
R=\left(\begin{array}{ll}
U & 0 \\
0 & U^{T^{-1}}
\end{array}\right), \quad \text { where } \operatorname{det} U \neq 0 ;
$$

(iii) semi-involutions:

$$
S=\left(\begin{array}{cc}
Q & I-Q \\
Q-I & Q
\end{array}\right),
$$

where $Q$ is a diagonal matrix of 0 's and 1's, so that $Q^{2}=Q,(I-Q)^{2}$ $=I-Q$.

Denote by $E_{i j}$ the $n$ by $n$ matrix with a 1 in the $i j$ th entry and zeros elsewhere. With $\alpha$ primitive in $G F(q)$, set

\footnotetext{
1 This research was supported in part under NSF G-9504.
} 


$$
D=\left(\begin{array}{cc}
\sum_{i=1}^{n-1} E_{i, i+1} & -E_{n 1} \\
E_{n 1} & \sum_{i=1}^{n-1} E_{i, i+1}
\end{array}\right)
$$

and

$$
J^{\prime}=\left(\begin{array}{cc}
I-\alpha E_{21} & 0 \\
0 & I+\alpha E_{12}
\end{array}\right) .
$$

It can be shown that for $q$ odd and $n>2, G(2 n, q)$ is the group generated by $D$ and $J^{\prime}$, i.e., every matrix of types (i), (ii), and (iii) is derivable from them.

Now, if we set

$$
J=\left(\begin{array}{cc}
I+\beta E_{12}-2 E_{22} & 0 \\
0 & I+\beta E_{21}-2 E_{22}
\end{array}\right)
$$

where $\beta=\alpha / 2$, then $J$ has period two and the group generated by $D$ and $J$ contains the matrix $J^{\prime}$.

For the case of $q$ even, re-define $J$ by

$$
J=\left(\begin{array}{cc}
I+\alpha E_{21} & E_{n n} \\
0 & I+\alpha E_{12}
\end{array}\right)
$$

where $\alpha$ is primitive in $G F\left(2^{m}\right)$. Then $J$ has period two, and the group generated by $D$ and $J$ is $G\left(2 n, 2^{m}\right)$, for $n>3$.

The group $G\left(6,2^{m}\right)$ can be generated by $D$ and the matrix

$$
J=\left(\begin{array}{cc}
I+\alpha E_{21} & \alpha^{-1} E_{33} \\
0 & I+\alpha E_{12}
\end{array}\right) \text {. }
$$

In the natural map of $G(2 n, q)$ onto $S(2 n, q)$, the matrices $D$ and $J$ are mapped onto generators, and the coset containing $J$ has period two.

The group $S(2, q)$ is the projective unimodular group, for which two generators are known (see [1]). Our method does not extend to the case $n=2$. However, the group $S(4,2)$ is isomorphic with $S_{6}$, the symmetric group on six letters, and so has two generators, one of period two.

In $[3 ; 4]$ Room and Smith have described a generation of the symplectic group over prime fields $G F(p)$ by two elements, one of which has period $p$. I am also informed by letter that Miss E. A. Whight of the University of Sydney has extended their theorem to include all finite fields. 


\section{REFERENCES}

1. A. A. Albert and J. G. Thompson, Two element generation of the projective unimodular group, Illinois J. Math. vol. 3 (1959) pp. 421-439.

2. L. E. Dickson, The theory of linear groups, New York, Dover (reprint), 1958.

3. T. G. Room, The generation by two operators of the symplectic group over $G F(2)$, J. Austral. Math. Soc. vol. 1 (1959) pp. 38-46.

4. T. G. Room and R. J. Smith, $A$ generation of the symplectic group, Quart. J. Math. vol. 9 (1958) pp. 177-182.

University of Chicago 